\title{
Selección en la diversidad de inhaladores: una actualización de bolsillo
}

\section{Selection in the diversity of inhalers: A pocket upgrade}

\author{
Rafael Bautista-Méndez,* Erika Salinas-Lezama, ${ }^{*}$ Liliana de Jesús Bonola-Gallardo,* \\ Rey David Pineda-Gudiño, ${ }^{*}$ Gerardo Ezequiel Magdaleno-Maldonado*
}

*Hospital Central Militar, Secretaría de la Defensa, Ciudad de México, México.

\begin{abstract}
RESUMEN. Hoy en día, la administración de medicamentos inhalados representa la ruta de elección para aplicar fármacos dirigidos a trastornos respiratorios. Para la correcta aplicación del tratamiento inhalado de los fármacos es fundamental la selección adecuada del dispositivo para cada paciente así como la revisión de la técnica de administración. Existen distintas consideraciones que deben ser tomadas en cuenta para lograr que la terapia inhalada sea óptima: conocer a profundidad los dispositivos disponibles, sus peculiaridades y técnica de administración así como las características clínicas, comorbilidades, edad, capacidad de inhalación del paciente. Existen inhalados que precisan un flujo inspiratorio alto para realizar una inhalación rápida y profunda, otros donde se debe realizar una inhalación lenta y constante, ya que no precisan un flujo alto. El objetivo de este artículo es realizar una revisión de los distintos inhaladores disponibles en la actualidad, con la presentación de los diferentes tipos de dispositivos, presentaciones, dosis y nombres comerciales en nuestro país para facilitar la práctica diaria a la hora de prescribirlos. De igual forma, recuerda al neumólogo la importancia de conocer las características de cada uno y la importancia de entender las necesidades y capacidades del paciente para poder elegir correctamente el dispositivo idóneo.
\end{abstract}

Palabras clave: Dosis medida, inhaladores, inhalador presurizado dosis medida, medicamentos inhalados, terapia respiratoria.

\section{INTRODUCCIÓN}

La administración de medicamentos inhalados representa la ruta de elección para aplicar fármacos dirigidos a la vía respiratoria en trastornos como asma y enfermedad pul-

Correspondencia:

\section{Dr. Rafael Bautista-Méndez}

Hospital Central Militar, Secretaría de la Defensa, México.

Correo electrónico: microrafa@hotmail.com

Trabajo recibido: 29-I-2020; aceptado: 24-IX-2020.

Citar como: Bautista-Méndez R, Salinas-Lezama E, Bonola-Gallardo LJ, Pineda-Gudiño RD, Magdaleno-Maldonado GE. Selección en la diversidad de inhaladores: una actualización de bolsillo. Neumol Cir Torax. 2020;79(3):204207. https://dx.doi.org/10.35366/96657
ABSTRACT. Today, the administration of inhaled medications represents the route of choice for applying drugs aimed at respiratory disorders. For the correct application of inhaled drug treatment, the proper selection of the device for each patient is essential, as well as the review of the administration technique. There are different considerations that must be taken into account to ensure that the inhaled therapy is optimal; To know in depth the available devices, their peculiarities and administration technique, as well as to take into account the clinical characteristics, comorbidities, age, inhalation capacity of the patient. There are inhales that require a high inspiratory flow to perform a rapid and deep inhalation, there are others where a slow and constant inhalation must be performed since they do not require a high flow. The goal of this narrative is to review the different inhalers currently available, with the presentation of the types of devices, doses and trade names in our country to facilitate daily practice when prescribing them. In the same way, it reminds the pulmonologist of the importance of knowing the characteristics of each one; and the importance of understanding the needs and capacities of the patient to be able to correctly choose the ideal device.

Keywords: Measured dose, inhalers, pressurized metered dose inhaler, inhaled medications, respiratory therapy.

monar obstructiva crónica. Entre estos medicamentos se encuentran los corticoesteroides, beta-agonistas, antagonistas muscarínicos. ${ }^{1}$ Las altas concentraciones de fármacos en los pulmones, pero bajas concentraciones sistémicas conferidas por inhalación, conducen a una alta eficacia, al tiempo que reducen el riesgo de efectos secundarios. ${ }^{2}$

Se ha reportado que $76 \%$ de los pacientes que usan inhaladores de cartucho presurizado y que de 49 a 54\% de los pacientes que usan inhaladores de sistema activado por inspiración cometen al menos un error cuando usan su inhalador. ${ }^{3}$ Además, entre 4 y $94 \%$ de los pacientes que usan inhaladores de polvo seco, no lo emplean correctamente y $25 \%$ de los pacientes nunca han recibido un entrenamiento para la técnica correcta de inhalación. ${ }^{4}$

Como profesionales de la salud es importante conocer las diferentes características y técnicas de administración 
que están disponibles para así poder elegir el más apropiado en cada paciente.

El objetivo de este artículo es realizar una narrativa de los distintos inhaladores disponibles en la actualidad, con la presentación de los diferentes tipos de dispositivos, presentaciones, dosis y nombres comerciales en nuestro país para facilitar la práctica diaria a la hora de prescribirlos. Como formación de neumólogo es crucial conocer las características de cada inhalador; y la importancia de entender las necesidades y capacidades del paciente para poder elegir correctamente el dispositivo idóneo.

\section{TERAPIA INHALADA}

La terapia inhalada utiliza la vía respiratoria para la administración de un fármaco, en forma de aerosol o suspensión de partículas microscópicas; el fármaco es liberado mediante un gas propelente que actúa como vehículo para llegar al tracto respiratorio. ${ }^{5}$ Debido a la complejidad del pulmón, existen múltiples procesos farmacocinéticos que son específicos del entorno pulmonar y de la ruta de inhalación, lo que hace que la farmacocinética pulmonar sea distinta y mucho más compleja que la de los medicamentos administrados por otras rutas. ${ }^{6}$ Dentro de los mecanismos farmacocinéticos se incluyen: el depósito de partículas/gotas de fármaco, disolución pulmonar del fármaco, aclaramiento mucociliar y de macrófagos, absorción al tejiido pulmonar, retención, metabolismo y eliminación del fármaco por absorción a la perfusión sistémica. ${ }^{2}$

El tamaño óptimo para que las partículas se depositen en los alvéolos y las vías respiratorias pequeñas es de 0.5-5 $\mu \mathrm{m}$. Las partículas más pequeñas están sujetas a sedimentación por gravedad. Éste es el mecanismo de depósito más efectivo en las vías respiratorias periféricas y se puede mejorar manteniendo la respiración después de la inhalación. ${ }^{7}$ También se ve modificado por el calibre y anatomía de la vía aérea, velocidad de emisión, volumen de aire inhalado, flujo inspiratorio, apnea posinhalación y técnica de inhalación. ${ }^{8}$

Tanto la dosis pulmonar como los patrones de depósito pulmonar dependen del tamaño aerodinámico de la partícula, el flujo inspiratorio, las características del dispositivo y los factores relacionados con la enfermedad, pero generalmente son independientes de las características fisicoquímicas del fármaco. ${ }^{9}$

Se han diseñado dispositivos de inhalación innovadores para emitir partículas pequeñas (diámetro aerodinámico de 1-5 $\mu \mathrm{m})$. En general, la inhalación más rápida da como resultado un aumento en el depósito en la región central, orofaringe, mientras que la inhalación más lenta da como resultado patrones de deposición más periféricos. La estrategia de diseño para el dispositivo de inhalación que combina el aerosol de movimiento lento con partículas de gotas más pequeñas se ha mostrado hasta ahora como el método más eficaz en términos de penetración distal de las vías respiratorias. ${ }^{10,11}$ La monocapa de células epiteliales de $0.2 \mu \mathrm{m}$ de grosor, que es mucho más delgada en comparación con las vías respiratorias conductoras, confiere mayor tasa de absorción en el espacio alveolar.,12

La mayoría de los inhaladores disponibles son heterodispersos, es decir, que las partículas del fármaco que producen son de distintos tamaños, los cuales siguen una distribución normal, desde $0.001 \mu \mathrm{m}$ hasta más de $100 \mu \mathrm{m}$ de diámetro. El valor que nos interesa como representativo del total, es el llamado diámetro de la mediana de la masa aerodinámica (DMMA), que es la medida de diámetro en la que la mitad de la masa de partículas tiene mayor tamaño y la otra mitad un tamaño menor. Otro concepto importante es la desviación estándar geométrica ( $\sigma \mathrm{g})$, que nos informa de la dispersión que presentan los diámetros de las distintas partículas. Con una og mayor de 1.2, hablamos de aerosoles heterodispersos, mientras que con una $\sigma \mathrm{g}$ menor de 1.2 se denominan monodispersos. ${ }^{13}$ Entre las características de los pacientes que deben tenerse en cuenta figuran la capacidad de coordinación, de aprendizaje y el grado de cumplimiento. ${ }^{14}$

\section{DISPOSITIVOS DE INHALACIÓN}

Existen tres grupos de dispositivos de inhalación: inhaladores de cartucho presurizado, inhaladores de vapor suave e inhaladores de polvo seco. ${ }^{5}$

Inhaladores cartucho presurizado o dosis medida (ICP o IDM; pMDI: pressurized metered dose inhaler). Utilizan un cartucho presurizado dosificador que contiene el fármaco activo en suspensión o solución con un gas propelente. Son multidosis y al accionarlos se libera una dosis controlada de fármaco. Se pueden utilizar con o sin cámaras de inhalación, ${ }^{13,14}$ pueden utilizarse con cámaras espaciadoras que presentan una válvula unidireccional antirretorno, que se abre con flujos inspiratorios bajos e impide el retorno del fármaco. Es importante mencionar que los IDM pueden presentar el efecto freón-frío por los propelentes (sensación de frío en la faringe cuando se inhala, lo que desencadena que se deje de inspirar debido a que provoca tos). ${ }^{8}$ Los $\mathrm{pMDI}$ se dividen en distintos tipos: ${ }^{13}$

- pMDI convencionales: fármaco disuelto en un gas, requiere sincronización precisa de inspiración/activación. Presentan bajo depósito pulmonar y un alto impacto orofaríngeo.

- pMDI de partículas extrafinas: proporcionan un aerosol de partículas más pequeñas, con mayor depósito pulmonar y menor impacto orofaríngeo.

- pMDI con sistema JET: tienen incorporado un espaciador circular de volumen muy pequeño y sin válvula unidireccional (equivalente a un pMDI convencional con cámara espaciadora). 
- BAI (Breath Actuated Inhaler) o sistema activado por inspiración: la válvula se activa con la inspiración del paciente, con autodisparo.

- Inhaladores de vapor suave (IVS; o de niebla fina o SMI: Soft Mist Inhaler): dispositivo que contiene una solución acuosa que genera vapor suave y libre de propelentes, el cual produce una nube fina de aerosol más lenta y con partículas de menor diámetro que los pMDI convencionales.

- Inhaladores de polvo seco (IPS; o DPI: Dry Powder Inhaler): surgen como opción adicional para solucionar los problemas de coordinación entre la activación y la

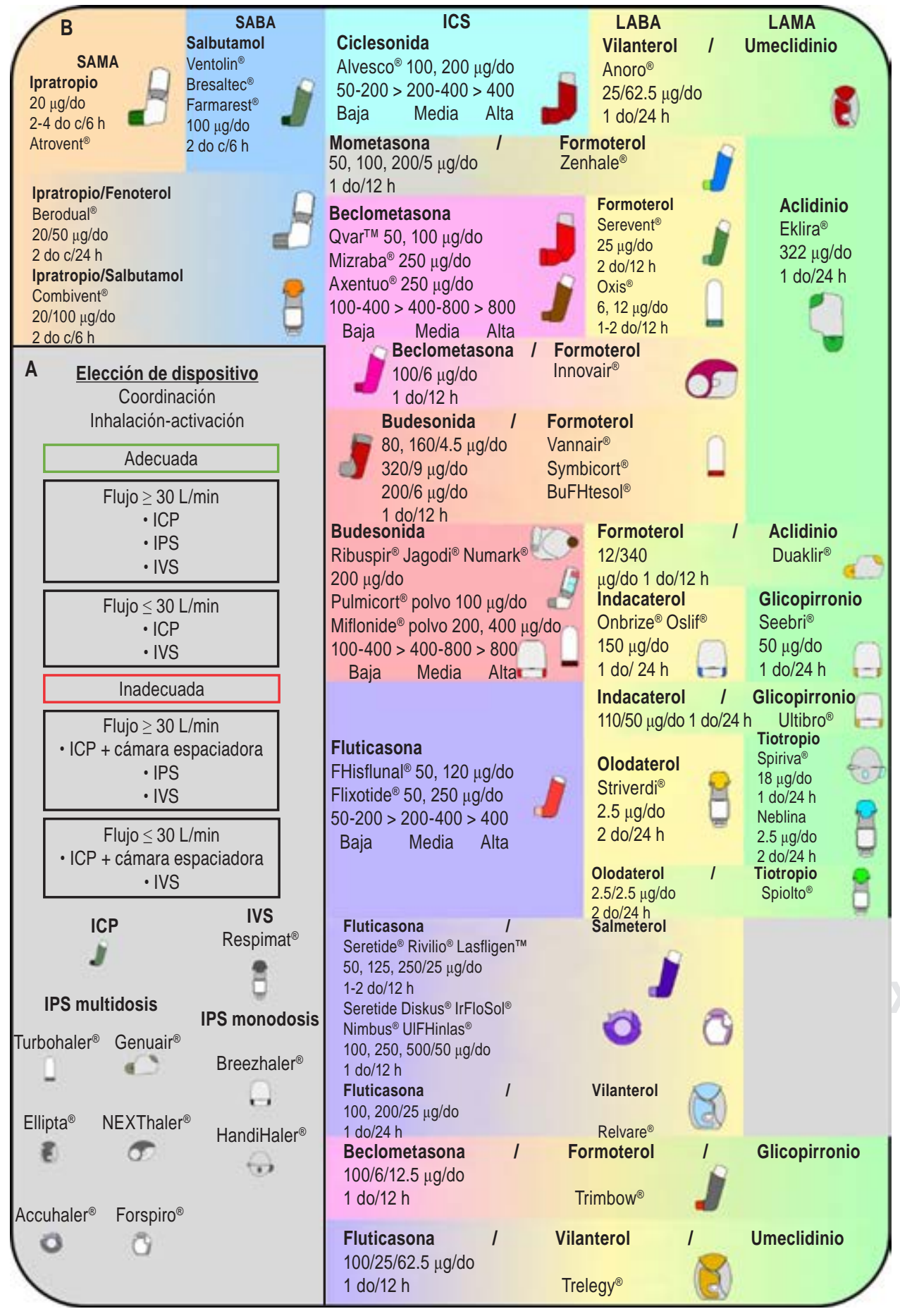

\section{Figura 1:}

Equivalencias ICS de acuerdo con la guía GUIMA 2017. A) Elección de dispositivo: coordinación inhalación-activación. B) Inhaladores.

SAMA = short-actin muscarinic antagonist (antagonista muscarínico de corta acción); SABA $=$ short-actin beta agonist (beta agonista de corta acción); LAMA = large-acting muscarinic antagonist (antagonista muscarínico de larga acción); $\mathrm{LABA}=$ long-acting beta agonist (beta agonista de larga acción) ICP = inhalador cartucho presurizado; IVS = inhalador vapor suave; IPS = inhalador polvo seco; ICS = corticosteroides inhalados; IVS = inhalador vapor suave.

Todos los nombres y marcas comerciales son propiedad de sus respectivos laboratorios. 
inspiración. Liberan polvo micronizado activado por el flujo inspiratorio del paciente, sin propelentes y adecuado depósito pulmonar. Requieren flujos inspiratorios más altos que los IDM. Tienen un depósito pulmonar superior al de otros sistemas (25-35\%).

- Sistemas predosificadores unidosis.

- Sistemas predosificadores multidosis.

Los IDM y los IVS son dispositivos basados en aerosol de baja resistencia, por lo que una inhalación lenta y constante es ideal para disminuir el depósito orofaríngeo y mejorar el suministro en los pulmones, sin requerir un flujo inspiratorio alto por parte del paciente. Los IPS presentan mayor resistencia del dispositivo, requieren una inhalación rápida y profunda, y flujos inspiratorios más altos (Figura 1A). ${ }^{5}$ En la Figura $1 B$ presentamos una recopilación de los inhaladores disponibles y sus distintas características con el fin de tener a la mano una herramienta sencilla al momento de seleccionar el tratamiento del paciente.

\section{CONCLUSIONES}

El objetivo de un dispositivo de inhalación es vehiculizar las partículas del fármaco, ya sea en suspensión, disolución o en forma de polvo seco hasta las zonas más distales del árbol respiratorio. Por ello, el tamaño de estas partículas (DMMA) debe encontrarse entre 0.5 y $5 \mu \mathrm{m}$, ya que por encima de ese rango se depositan por impactación en la vía aérea superior sin que progresen, y por debajo vuelven a exhalarse por difusión sin haberse absorbido. Todos los dispositivos del mercado producen partículas dentro de estos tamaños, lo que las caracterizan, aparte del dispositivo, es la fuerza que las mueve hasta el árbol bronquial. ${ }^{13}$ De aquí la importancia de conocer las características de cada uno y la importancia de entender las necesidades y capacidades del paciente para poder elegir correctamente el dispositivo idóneo. ${ }^{15,16}$

\section{REFERENCIAS}

1. Lavorini F, Mannini C, Chellini E. Challenges of inhaler use in the treatment of asthma and chronic obstructive pulmonary disease. EMJ Respir. 2015;3(2):98-105.

2. Markus-Borghardt J, Kloft C, Sharma A. Inhaled therapy in respiratory disease: the complex interplay of pulmonary kinetic processes. Can Respir J. 2018;2018:2732017. Available in: https:// doi.org/10.1155/2018/2732017
3. Molimard M, Raherison C, Lignot S, Depont F, Abouelfath A, Moore N Assessment of handling of inhaler devices in real life: an observational study in 3811 patients in primary care. J Aerosol Med. 2003;16(3):249254. Available in: https://doi.org/10.1089/089426803769017613

4. Lavorini F, Magnan A, Dubus JC, Voshaar T, Corbetta L, Broeders M, et al. Effect of incorrect use of dry powder inhalers on management of patients with asthma and COPD. Respir Med. 2008;102(4):593-604. Available in: https://doi.org/10.1016/j.rmed.2007.11.003

5. Usmani OS. Choosing the right inhaler for your asthma or COPD patient. Ther Clin Risk Manag. 2019;15:461-472. Available in: https:// doi.org/10.2147/tcrm.s160365

6. Weber B, Hochhaus $\mathrm{G}$. A pharmacokinetic simulation tool for inhaled corticosteroids. AAPS J. 2013;15(1):159-171. Available in: https://doi. org/10.1208/s12248-012-9420-z

7. Tsuda A, Henry FS, Butler JP. Particle transport and deposition: basic physics of particle kinetics. Compr Physiol. 2013;3(4):1437-1471. Available in: https://doi.org/10.1002/cphy.c100085

8. Dispositivos de inhalación para asma y EPOC. Bol Ter ANDAL. 2018;33(2):9-19. Disponible en: $h$ ttp://dx.doi.org/10.11119/BTA2017-33-02

9. Labiris NR, Dolovich MB. Pulmonary drug delivery. part I: physiological factors affecting therapeutic effectiveness of aerosolized medications. Br J Clin Pharmacol. 2003:56(6):588-599. Available in: https://doi. org/10.1046/j.1365-2125.2003.01892.x

10. Bonini M, Usmani OS. The importance of inhaler devices in the treatment of COPD. COPD Res Pract. 2015;1(9). Available in: https:// doi.org/10.1186/s40749-015-0011-0

11. Thompson PJ. Drug delivery to the small airways. Am J Respir Crit Care Med. 1998:157(5 Pt 2):S199-S202. Available in: https://doi. org/10.1164/ajrccm.157.5.rsaa-7

12. Olsson B, Bondesson E, Borgström L, Edsbäcker S. Pulmonary drug metabolism, clearance, and absorption. In: Controlled pulmonary drug delivery. New York, NY: Springer; 21-50. Available in: https:// www.researchgate.net/deref/http\%3A\%2F\%2Fdx.doi.org\%2F 10.100 7\%2F978-1-4419-9745-6_2

13. Consenso SEPAR-ALAT sobre terapia inhalada. Arch Bronconeumol. 2013;49(Supl 1):2-14. Disponible en: https://doi.org/10.1016/S03002896(13)70068-1

14. Cases GS, Aragonés C, Toya AA, Carreño GM, Peiró MJF. Dispositivos y guía de administración vía inhalatoria. Rev OFIL. 2017;27(1):31-46.

15. Sanchis J, Gich I, Pedersen S; Aerosol Drug Management Improvement Team (ADMIT). Systematic review of errors in inhaler use: has patient technique improved over time? Chest. 2016;150(2):394-406. Available in: https://doi.org/10.1016/j.chest.2016.03.041

16. Miravitlles M, Soler-Cataluña JJ, Alcázar B, Viejo JL, Gracía-Río F. Factors affecting the selection of an inhaler device for COPD and the ideal device for different patient profiles. Results of EPOCA Delphi consensus. Pulm Pharmacol Ther. 2018;48:97-103. Available in: https://doi.org/10.1016/j.pupt.2017.10.006

Conflicto de intereses: Los autores declaran no tener conflicto de intereses. 